\title{
Ultrasonographic and biochemical assessments as early prediction of polycystic ovarian syndrome in obese women
}

\author{
Alaa A Ahmed ${ }^{1}$, Said S Moselhy ${ }^{2}$, Taha A Kumosani ${ }^{3}$, Etimad A Huwait ${ }^{3}$, Maryam A AL-Ghamdi ${ }^{3}$, \\ Khalid A AL-Madani ${ }^{4}$, Majdi H AlToukhi ${ }^{5}$, Afnan T Kumosani ${ }^{6}$
}

1. Head of Radiology Department, Ghamra Armed Forces Hospital, Cairo-Egypt.

2. Biochemistry Department, Faculty of Science, Ain Shams University, Cairo, Egypt.

3. Biochemistry Department, Faculty of science, King Abdulaziz University, Jeddah. Experimental Biochemistry unit, King Fahd Medical Research center and Production of bio products for industrial applications research Group, King Abdulaziz University.

4. Consultant in Clinical Nutrition,Member of the Boardof ILSI Middel East, Member of the Board of Saudi Society for Food and Nutrition. Advance Clinics, Jeddah, Saudi Arabia.

5. Consultant Public Health \& Infectious Diseases, Director Health \& Environment Department, The General Authority of Metrology and Environmental Protection, Saudi Arabia.

6. Department of pathology and Laboratory Medicine, Transfusion Blood medicine Bank, King Abdulaziz Medical city, Jeddah, National Guards, , Jeddah, 21423.

\begin{abstract}
Backgroud: Polycystic ovary syndrome (PCOS) is considered as a common cause of hormonal disturbance and obesity. The diagnosis of PCOS was done by different methods including clinical signs as anovulation, hyperandrogenism, biochemical markers and ultrasounographic investigation. This study investigated comparative outcomes of ultrasonographic and biochemical markers for early prediction of PCOS in obese women.

Subjects and methods: Seventy-five patients were clinically diagnosed with obese, PCOS and obese with PCOS and twenty-five normal age matched subjects were enrolled as control. Abdominal and transvaginal ultrasonographic for assessment of ovarian properties. In addition, BMI, serum free testosterone, dehydroepiandrosterone (DHEA), insulin, glycosylated hemoglobin (HbA1c) and LDL-c levels were evaluated.

Results: In obese patients with PCOs $(20 \%)$ ovaries revealed normal appearance in morphology while the rest $(80 \%)$ showed PCOs in the form of cysts of $2-8 \mathrm{~mm}$ in diameter peripherally arranged around stroma. A significant elevation of free testosterone, DHEA and insulin in obese with or without PCOS compared with obese group $(\mathrm{p}<0.001)$. A positive correlation with hormonal abnormalities of increased HA1c, LDL-c, free testosterone, DHEA and insulin compared with obese only.

Conclusion: According to our study findings, ovarian morphology combined with biochemical markers is more reliable for early prediction and diagnosis of PCOS for interpretation and management.

Keywords: PCOS; Ultrasound; Diagnosis; hormones.

DOI: https://doi.org/10.4314/ahs.v20i2.18

Cite as: Abmed AA, Moselhy SS, Kumosani TA, Huwait EA, AL-Ghamdi MA, AL-Madani KA, et al. Ultrasonographic and biochemical assessments as early prediction of polycystic ovarian syndrome in obese women. Afri Health Sci. 2020; 20(2): 676-681. https:/ / doi. org/10.4314/abs.v20i2.18
\end{abstract}

\section{Introduction}

The Polycystic ovary syndrome (PCOS) is considered as a common disturbance of sexual hormonal system ${ }^{1}$.

\section{Corresponding author:}

Said S Moselhy,

Biochemistry Department, Faculty of Science,

Ain Shams University, Cairo, Egypt.

Email: moselhy6@hotmail.com
The diagnosis of PCOS was done by different methods including chronic anovulation, hyperandrogenism and by ultrasonography. PCOS is the majority causes of female infertility which is a risks factor of metabolic syndrome, diabetes mellitus, cardiovascular diseases and some cancer ${ }^{3}$. It was proved that, insulin resistance and hyper-insulinemia play a role in the incidence of PCOS $^{4}$. The insulin resistance in PCOS was higher in obese women without $\mathrm{PCOS}^{5}$ and is a common feature of PCOS in both normal and obese women. Insulin re-
(C) 2020 Ahmed AA et al. Licensee African Health Sciences. This is an Open Access article distributed under the terms of the Creative commons Attribution License (https://creativecommons.org/licenses/BY/4.0), which permits unrestricted use, distribution, and reproduction in any medium, provided the original work is properly cited. 
sistance also found to play an important role in the development of the infertility of PCOS and hyperandrogenism ${ }^{6}$. Women with dysmenorrhea, insulin resistance and hyperandrogenism are at high risk of developing $\operatorname{PCOS}^{7}$. It was found that, hyperinsulinemia associated with increased risk of cardiovascular disease directly correlated to lipid metabolism abnormality ${ }^{8}$. The PCOS is a disorder caused by heterogeneous factors with variable manifestations ${ }^{3}$. The diagnosis of PCOS depends on the combination of clinical and laboratory investigation of specific sexual hormones including serum luteinizing hormone, testosterone, and reduction of sex hormone binding globulin?

Swanson et al. ${ }^{10}$ reported that, the ovary of PCOS showed enlarged and rounded, with a volume of $12 \mathrm{~cm}$ and high number of follicles $(2-8 \mathrm{~mm})$ encircling the ovarian cortex. However, ovarian size showed a significant overlap between normal and PCOS ovaries and limit normal has reduced from 1.0 to $5.0 \mathrm{~cm}^{11}$. Adams et al. ${ }^{12}$ stated that, trans abdominal ultrasound, showed 10 or more cysts of $2-7 \mathrm{~mm}$ arranged around an echo dense stromal. The assessment and management of PCOS provides a good index guidelines to physician on evidence based diagnosis and management ${ }^{13}$.

The evaluation of expected PCOS is twofold: to exclude other treatable conditions that can confers to PCOS and to detect and treat long-term metabolic complications. Anovulation is common after menarche, so it is reasonable to delay workup for PCOS in adolescents . If an adolescent is diagnosed for PCOS, it was expected that she meet three of the Rotterdam criteria including irregular menstrual cycles and hirsutism, signs of hyperandrogenism and a constellation of oligomenorrhea, amenorrhea, or infertility

The current study hypothesized that ovarian morphology combined with biochemical markers in obese with or without PCOS could be relevant to its volume as a good index for early predication of disease for fast management and interpretation.

\section{Subjects and methods \\ Subjects}

This study was carried out according to Ethical approval committee of Ain Shams University, Egypt .Studied subjects were medically examined at the Gynecology clinic. A consent formal was taken by all subjects. A hundred women aged 20-40 years, were included in this study divided into four groups; Group I: Normal healthy women with BMI (20-22); group II: Obese women with BMI $(>30)$; group III: non obese with PCOS with BMI (21-22.5); diagnosed by transvaginal ultrasonography and Group IV: Obese with PCOS BMI (>30). Clinical symptoms of PCOs including irregular menstrual cycles and hirsutism, signs of hyperandrogenism and a constellation of oligomenorrhea, amenorrhea, or infertility ${ }^{14}$.

Fasting 12 hours blood sample were collected from all subjects. Serum samples were subjected for analysis of free testosterone, dehydroepiandrosterone (DHEA), insulin, Glycosylated hemoglobin (HbA1c) and LDL-c. The exclusion criteria including subjects on contraception, fertility medications or abnormal cortisol or thyroid hormones.

This current study included a sample size of 100, based on available resources rather than power analysis

\section{Ultrasound investigation}

The ultrasound instrument was a Voluson E6 (GE Healthcare, Zipf, Austria), a 2-5 MHz abdominal probe and a $7.5-\mathrm{MHz}$ transvaginal probe, all scans were performed in a private room after getting patient consent. Ultrasound investigation was done from 3th Day to 7 th of the menstrual cycle. Using abdominal probe and transvaginal probe. Each ovary was scanned and anatomic observed with respect to the utero-ovarian ligament. The parameters should include (1) volume, (2) number of follicle (3) diameter of each ovary.

\section{Baseline examination}

All subjects were evaluated by taking history, clinical investigation and were to check for general conditions. Patients were evaluated for hirsutism at baseline and at the end of the study using the modified Ferriman-Gallwey $(F-G)$ score 15 . The value of F-G that are 8 or greater was considered to be hirsute. Abdominal and Ultrasound were done to confirm diagnosis of PCOS.

\section{Statistical analysis}

Statistical analysis were performed using the Statistical Package for Social Sciences (SPSS) vs. 20. The data were summarized using means and standard deviations and ranges, categorical data were summarized as numbers and percentages. Comparisons between the 2 groups with respect to normally distributed numeric variables were done using independent t-test. All p-values were two-sided. P-values $<0.05$ were considered significant. 
Results were statistically analyzed using SPSS program version 20. $\mathrm{p}$ value $<0.05$ considered as significance.

\section{Results}

Demographic analysis including weight and BMI was shown in table (1). The study includes four groups of subjects with the age range between 20 and 40 (average of 31 years). PCOS patients were $(30 \%)$ suffered from irregularities of menstrual cycle, $(30 \%)$ with infertility, $(20 \%)$ with obesity and $(20 \%)$ with hirsutism.

Table 1: Anthropometric parameters of all studied groups.

\begin{tabular}{|c|c|c|c|c|c|}
\hline \multirow[b]{2}{*}{ Variables } & \multicolumn{2}{|c|}{ Non obese } & \multicolumn{2}{|c|}{ Obese } & \multirow[b]{2}{*}{ P-Value } \\
\hline & $\begin{array}{l}\text { Normal ovary } \\
\quad(n=25)\end{array}$ & $\begin{array}{l}\text { PCOs } \\
(\mathrm{n}=25)\end{array}$ & $\begin{array}{c}\text { Normal ovary } \\
\quad(n=25)\end{array}$ & $\begin{array}{l}\text { PCOs } \\
(\mathrm{n}=25)\end{array}$ & \\
\hline $\begin{array}{c}\text { Weight } \\
\text { (Kg) }\end{array}$ & $75.73 \pm 6.97$ & $69.26 \pm 23.44$ & $107.45 \pm 8.82$ & $100.61 \pm 11.27$ & $\mathrm{p}<0.05$ \\
\hline BMI & $22.77 \pm 2.06$ & $21.48 \pm 1.6$ & $28.19 \pm 2.05$ & $29.37 \pm 7.19$ & $\mathrm{p}<0.05$ \\
\hline
\end{tabular}

Data in fig.1 showed that, a significant elevation of without PCOS compared with obese group $(\mathrm{p}<0.05)$. glycosylated hemoglobin $(\mathrm{p}<0.05)$, LDL-c, free testos- In addition, these parameters were significantly elevated terone $(p<0.05)$, DHEA and insulin in obese with or in obese $(p<0.05)$ versus control group.

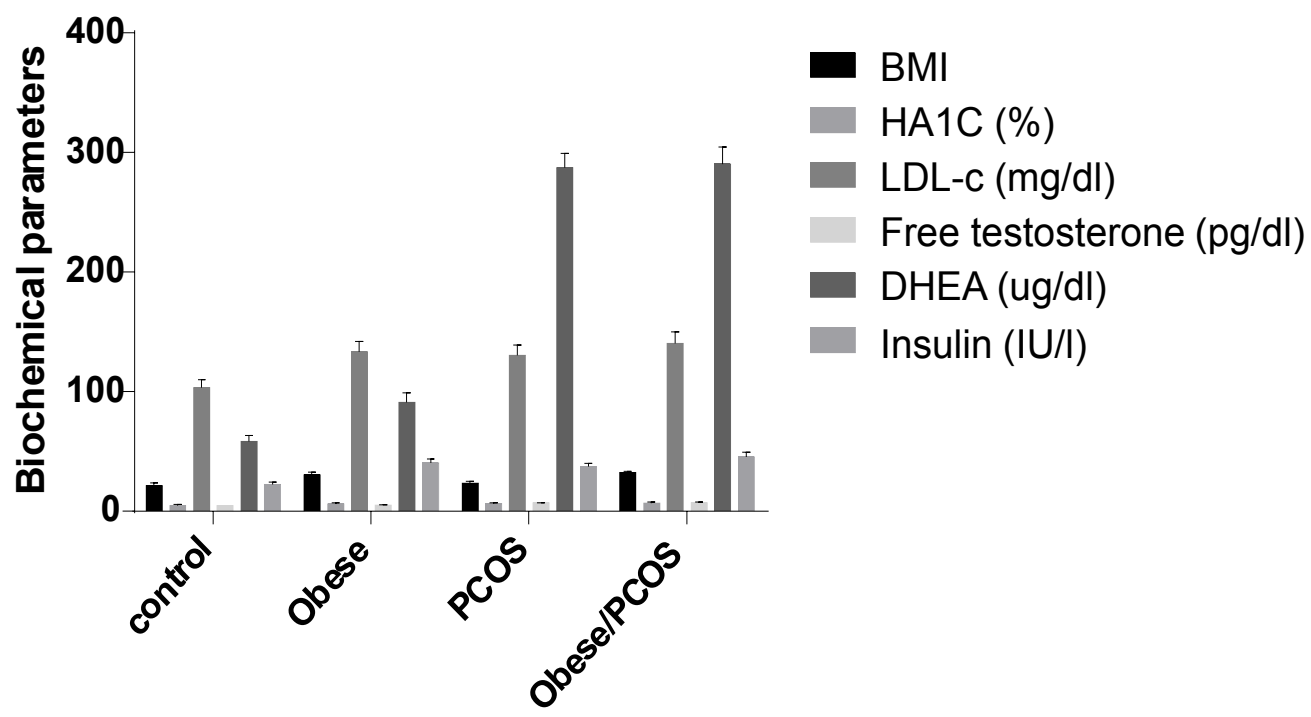

\section{Studied groups}


Results in Fig (2) showed ovarian volume in different studied groups. In control group, ovarian volume was $(9.1 \mathrm{ml})$, obese $(9.4 \mathrm{ml})$, PCOS $(11.2 \mathrm{ml})$ and obese with
PCOS $(11.1 \mathrm{ml})$. The ovarian volume was statistically higher in obese with PCOs than non-obese $(p<0.05)$. However, $20 \%$ of patients showed a normal ovarian volume $(\mathrm{p}>0.05)$.

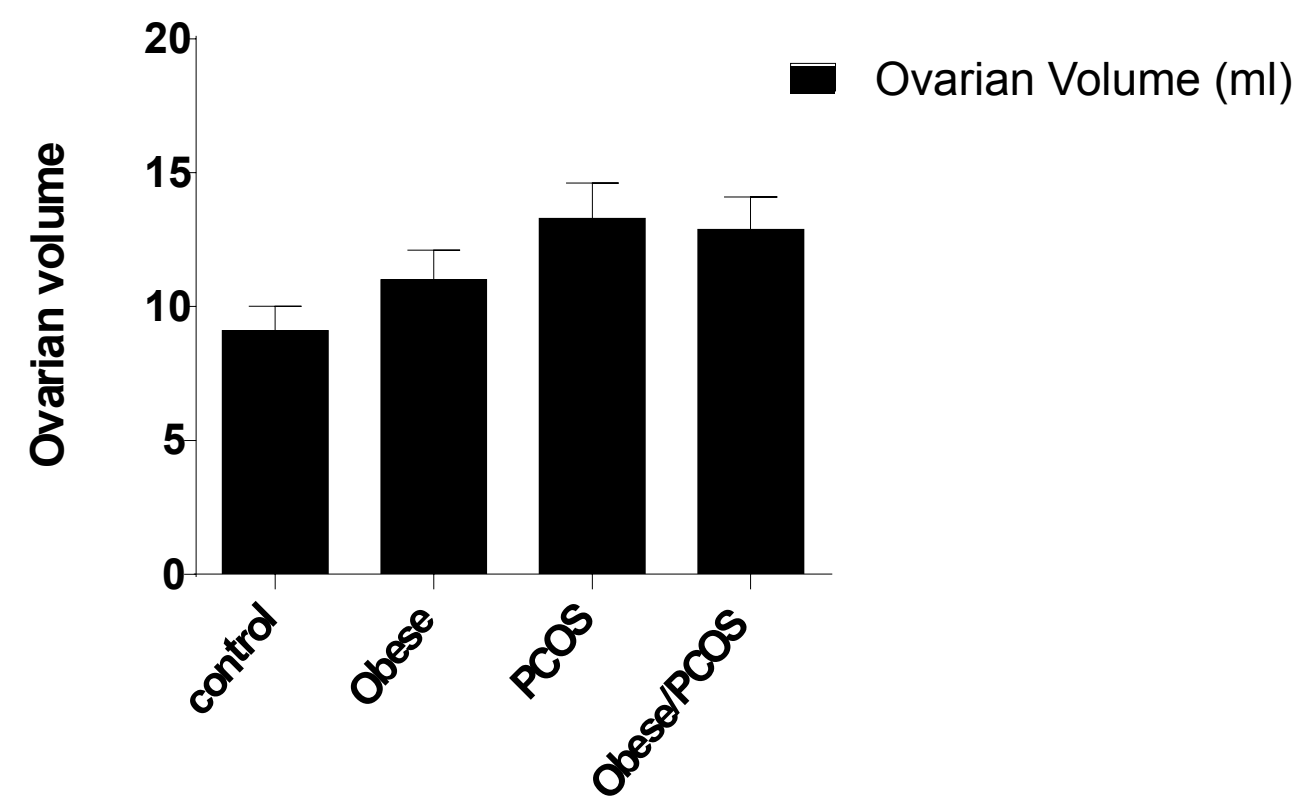

Fig 2. Studied groups

Serum DHEA and insulin were significantly higher obese with PCOs than PCOs $(\mathrm{p}<0.05)$.

The morphology of ovary was determined based on the number of follicle count, follicle size diameter and its distribution. In obese with PCOS 33\% showed normal morphological pattern ( $p>0.05)$.wile $(67 \%)$ showed a picture of PCOS more than 10 cysts in $2-8 \mathrm{~mm}$ in diameter arranged around an echodense stroma (Fig 3).

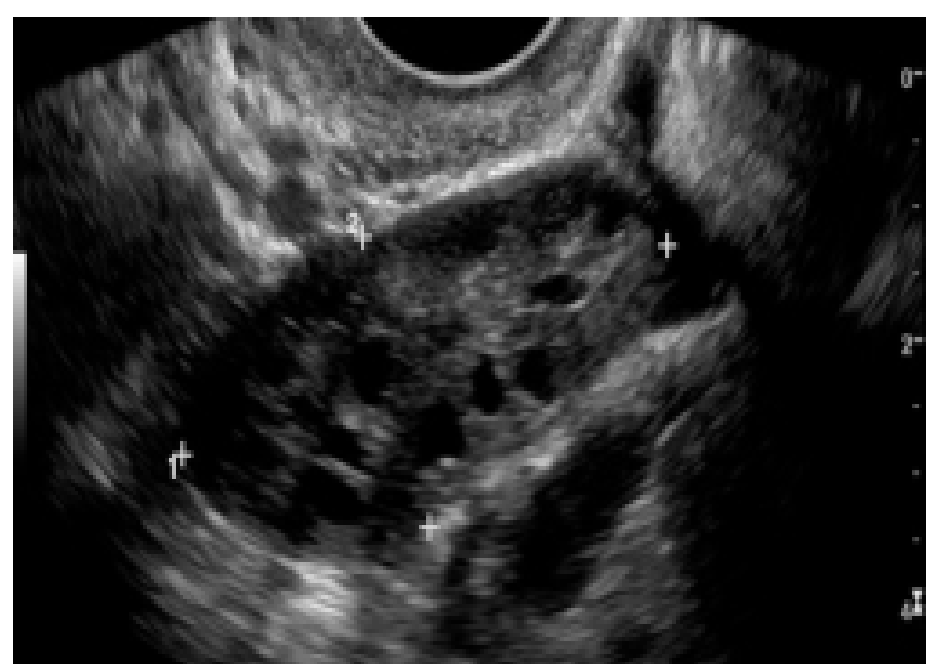

Fig. 3: A female with PCO, showing appearance of multiple follicles peripherally arranged peripherally on dense stroma. 


\section{Discussion}

The PCOS is an endocrine disorder of unknown cause $^{14}$. The PCOS should meet these criteria: anovulation; hyperandrogenism and ultrasound investigation ${ }^{15}$. Ultrasonographic is a non-invasive technique used for evaluating and diagnosis of different diseases including PCOS. This study was proposed to assess a sensitive and specific markers used in diagnosis and prognosis of diseases. For that both techniques were applied for more precise identification of PCOS. Obesity is the major cause of public health problem and contributes to many diseases such as metabolic syndrome, CVD, hypertension and PCOS ${ }^{14}$. The diagnosis of PCOS is not enough since in some cases ovary appears as normal, for that, additional to clinical as biochemical investigations are required for early prediction and diagnosis of PCOS for early interpretation and management. Clinical diagnosis of PCOS includes oligo - or anovulation. In current study it ws found that, obese women with or without PCOS showed elevated insulin level and resistance, elevated LDL-C level, free testosterone and DHEA. The criteria of PCOs including $\geq 12$ follicles in (2-9) $\mathrm{mm}$; a peripheral arrangement of ovarian follicles; its volume $>10 \mathrm{~cm}$ and a highly echogenic .

Our data in agreement with Lujan et al. ${ }^{15}$ who reported that a higher threshold than 12 is required to adequately differentiate between PCOs and normal ovaries ${ }^{16}$.

In this study data obtained showed that, clinically and biochemical analysis confirm PCOS showed morphological changes by ultrasonographic investigation in $80 \%$./span $>$ In $20 \%$ of patients, they showed normal ovary appearance with abnormal biochemical markers. The data obtained revealed that 12 or more follicles were observed. This results in agreement with Lujan et al. ${ }^{15}$ who reported that a significant elevation than 12 is enough to differentiate between PCOS and normal. Biochemical changes were observed in $100 \%$ of patients with obese and PCOS. The cutoff of $>10 \mathrm{~cm}$ was associated with $99.0 \%$ specificity, but only $75 \%$ sensitivity, in differentiating between normal and $\mathrm{PCOS}^{16,17}$. A lower threshold of $7 \mathrm{~cm}$ and a higher threshold of 13 $\mathrm{cm}$ suggested as being more appropriate thresholds for PCOS morphology.

Clinicians and researchers use a myriad techniques ranging from semi-automated to manual calculations using linear measurements. In the present study, we used the equation for a prolate spheroid, rather than equation of a prelate ellipsoid which is better with volume assessment of PCOS made by 3D ultrasound ${ }^{18}$. We employed the biochemical investigation, rather than the commonly used ultrasonography investigation, since this method was found to correlate better with measurements of PCOS made by ultrasonography ${ }^{19}$.

Difference between obese and non-obese women with PCOS in serum hormone levels, especially with regard to free testosterone and DHEA. Obesity showed an elevation in free Testosterone in women with PCOS compared with non -obese, this is in accordance with study of Mora et $\mathrm{al}^{20}$. It was found that, Insulin was elevated significantly in obese PCOS patients compared with non-obese. This interpretation can be in counting significant in diagnosis and prognosis of PCOS.

\section{Conclusion}

Hormonal changes in combination with clinical symptoms increased the accuracy and sensitivity of ultrasound side by side ( $>10$ follicles of $8 \mathrm{~mm}$ ) diameter is found in patients included in the study in controversy to enlargement of ovarian volume and more reliability of using biochemical in combination with ultrasound for ovarian volume and morphology in early diagnosing of subjects suspected to develop PCOS for control and management.

\section{Conflict of interest}

There is no conflict of interest to declare.

\section{Limitation of the study}

No limitations are found. The strength of study depend on the sensitive biomarkers support ultrasonography for accurate diagnosis of PCOs.

\section{References}

1. Lam MR and Johnson JR. Three-dimensional ultrasound features of the polycystic ovary and the effect of different phenotypic expressions on these parameters. Hum Reprod, 22 (12) (2007), pp. 3116-3123CrossRef.

2. Lam P, Rainefenning M, Cheung L, Haines C. Three-dimensional ultrasound features of the polycystic ovary in Chinese women. Ultrasound Obstet Gynecol (2009): 34; 196-200. published online in Wiley Inter Science.http://dx.doi.org/10.1002/uog.6442.

3. Franks S. Polycystic ovary syndrome: a changing perspective. Clin Endocrinol (Oxf), 31 (1989), pp. 87-120

4. Sasha JM, Aleksandra AB, Iskra B, Irfan A, Biljana T, Gordana P, Tatjana M, Brankica K. Indexes of Insulin Resistance in Hyperinsulinemic Polycystic Ovary Syndrome in a Macedonian Cohort of Women of Reproductive Age: A Cross-Sectional Study. Open Ac- 
cess Macedonian Journal of Medical Sciences. 2016 Dec 15; 4(4):607-612.

5. ConwayJ and Honour HJ. Heterogeneity of the polycystic ovary syndrome: clinical, endocrine and ultrasound features in 556 patients Clin Endocrinol (Oxf), 30 (1989), pp. 459-470

6. The Rotterdam ESHRE/ASRM-Sponsored PCOS consensus workshop group. Revised 2003 consensus on diagnostic criteria and long-term health risks related to polycystic ovary syndrome (PCOS). Hum Reprod. 2004; 19: 41-47.

Google Scholar

7. Orsini L, Venturoli S, Lourusso R, Pichinotta V, Paradisi R,Bovicelli L. Ultrasound findings in polycystic ovarian disease. J Ultrasound Med, 4 (1985), pp. 341-351 . 8. Nicolini U E, Ferrazzi M, Bellotti P, Travaglini R, Elli RS. The contribution of sonographic evaluation of ovarian size in patients with polycystic ovarian disease. $J$ Ultrasound Med, 4 (1985), pp. 347-351.

9. Robert L Rosenfield, David A Ehrmann. The Pathogenesis of Polycystic Ovary Syndrome (PCOS): The Hypothesis of PCOS as Functional Ovarian Hyperandrogenism Revisited. Endocrine Reviews. 2016; 37(5): $467-$ 520.

10. Swanson E, Sauerbrei PC. Medical implications of ultrasonically detected polycystic ovaries. J Clin Ultrasound, 9 (1981), pp. 219-222.

11. Lakhani K W, Purcell R, Fernando PH. Ovarian volume and polycystic ovaries. Eur J Ultrasound, 7 (1998), pp. S21-S22

12. Adams JS, Franks D, Polson H, Maso N, Abdulwahid $\mathrm{M}$ et al. Multifollicular ovaries: clinical and endocrine features and response to pulsatile gonadotropin releasing hormone. Lancet, 2 (1985), pp. 1375-1379

13. Robert YF, Dubrulle L, GaillandreY. Ardaens, P. Thomas-Desrousseaux L, Lemaitre et al. Ultrasound assessment of ovarian stroma hypertrophy in hyperandrogenism and ovulation disorders: visual analysis versus computerized quantification Fertil Steril, 64 (1995), pp. 307-312

14. Tracy W and Rami M. Diagnosis and Treatment of Polycystic Ovary Syndrome. Am Fam Physician. 2016\&\#a0;Jul 15;94(2):106-113.

15. Lujan E, Chizen R, Peppin K, Dhir A, Pierson A. Assessment of ultrasonographic features of polycystic ovaries is associated with modest levels of inter-observer agreement. J Ovarian Res, 2 (2009) 6-10.

16.Guraya S. Prevalence and ultrasound features of polycystic ovaries in young unmarried Saudi females. $J$ Microsc Ultrastruct, 1 (1) (2013), pp. 30-34

17. Wild RA, Carmina E, Diamanti-Kandarakis E, Dokras A, et al. Assessment of cardiovascular risk and prevention of cardiovascular disease in women with the polycystic ovary syndrome: a consensus statement by the Androgen Excess and Polycystic Ovary Syndrome (AE-PCOS) Society. J Clin Endocrinol Metabol, 95 (5) (2010), pp. 2038-2049

18. Allemand M, Tummon I, Phy J, Foong S, Dumesic D, Session D. Diagnosis of polycystic ovaries by three-dimensional transvaginal ultrasound. Fertil Steril, 85 (1) (2006), pp. 214-219.

19. Allemand M, Tummon I, Phy J, Foong S, Dumesic D, Session D. Diagnosis of Polycystic ovaries by Three-Dimensional Transvaginal Ultrasound Fertil Steril, 85 (1) (2006), pp. 214-219.

20. Mora MM, María I, Elena F, José L. San-Millán, Héctor F. Escobar-Morreale Effects of Polycystic Ovary Syndrome (PCOS), Sex Hormones, and Obesity on Circulating miRNA-21, miRNA-27b, miRNA-103, and miRNA-155 Expression. The Journal of Clinical Endocrinology \& Metabolism, Volume 98, Issue 11, 1 November 2013, Pages E1835-E1844, https://doi.org/10.1210/ jc.2013-2218. 